\title{
Discrete-time modeling and control for the differential power processing PV architecture based on DAB converters
}

\author{
Xiaodong $\mathbf{L u}^{\mathrm{a})}$ \\ School of Instrumentation Science and Opto-electronics Engineering, \\ Beihang University, 37 Xueyuan Road, Haidian District, Beijing 100191, China \\ a)xiaodong.lu@buaa.edu.cn
}

Abstract: A differential power processing (DPP) isolated-port photovoltaic (PV) architecture based on dual active bridge submodule integrated converters (subMICs) is designed to improve the energy capture efficiency of a PV system in the presence of mismatch conditions. To facilitate the control design, a small-signal model of the DPP PV architecture is developed in this work based on the discrete-time modeling method. Discrete compensator for the subMICs is designed accordingly. The validity of the modeling and control is verified by experimental results.

Keywords: differential power processing (DPP), isolated-port PV architecture, dual active bridge (DAB), discrete-time modeling

Classification: Electron devices, circuits, and systems

\section{References}

[1] C. Olalla, D. Clement, M. Rodriguez and D. Maksimovic: IEEE Trans. Power Electron. 28 (2013) 2980. DOI:10.1109/TPEL.2012.2219073

[2] T. Esram and P. Chapman: IEEE Trans. Energy Convers. 22 (2007) 439. DOI:10.1109/TEC.2006.874230

[3] S. Idenoue and T. Sato: IEICE Technical Report 114 (2014) 17.

[4] G. R. Walker and P. C. Sernia: IEEE Trans. Power Electron. 19 (2004) 1130. DOI:10.1109/PSEC.2002.1023842

[5] R. Pilawa-Podgurski and D. Perreault: IEEE Trans. Power Electron. 28 (2013) 2957. DOI:10.1109/TPEL.2012.2220861

[6] P. Shenoy, K. Kim, B. Johnson and P. Krein: IEEE Trans. Power Electron. 28 (2013) 2968. DOI:10.1109/TPEL.2012.2211082

[7] D. Costinett, K. Hathaway, M. Rehman, M. Evzelman, R. Zane, Y. Levron and D. Maksimovic: IEEE Appl. Power Electron. Conf. Expo. (2014) 3230. DOI:10.1109/APEC.2014.6803768

[8] D. J. Packard: Ph.D. dissertation California Institute of Technology (1976).

[9] A. R. Brown and R. D. Middlebrook: IEEE Power Electron. Spec. Conf. (1981) 349. DOI:10.1109/PESC.1981.7083659

[10] D. Maksimovic and R. Zane: IEEE Trans. Power Electron. 22 (2007) 2552. DOI:10.1109/TPEL.2007.909776

[11] M. Sato and Y. Ishizuka: IEICE Technical Report 114 (2015) 13. 
[12] R. Ahmadi, D. Paschedag and M. Ferdowsi: 36th Ann. Conf. IEEE Ind. Electron. Soc. (2010) 2311. DOI:10.1109/IECON.2010.5675123

\section{Introduction}

It is well known that mismatches due to partial shading, manufacturing tolerances, dirt, or aging result in significant losses in the energy capture of a photovoltaic (PV) system. To solve this issue, a differential power processing (DPP) isolated-port PV architecture has been proposed [1]. The structure of this architecture is shown in Fig. 1(a). A PV module is typically divided into several submodules, each of which consists of a string of PV cells bypassed by a backplane diode. The input of each submodule integrated converter (subMIC) is connected directly to a PV submodule, while all outputs of the subMICs are connected in parallel as the isolated port. Each subMIC is controlled to equalize its input voltage, the submodule voltage $v_{s u b_{i}}$, to the isolated-port voltage $v_{\text {port }}$, which results in equal submodule voltages. Since all the submodules are connected in series, identical module current $i_{\text {mod }}$ passes through the submodules. Consequently, differential currents $i_{s u b_{i}}$ between the submodule currents $i_{p v_{i}}$ and $i_{\text {mod }}$ are processed by the subMICs and converge at the isolate port, which means $i_{s u b_{i}}=i_{p v_{i}}-i_{\text {mod }}$ and $\sum i_{s u b_{i}}=0$. As a result, module current $i_{\text {mod }}$ is equal to the average value of submodule currents $i_{p v_{i}}$.

Fig. 1(b) shows the effect of the DPP isolated-port PV architecture when submodule short-circuit currents $I_{s c_{1,2}}=4 \mathrm{~A}, I_{s c_{3}}=2.5 \mathrm{~A}$. In this case, submodule 3 is mismatched by $3 / 8$. Without the DPP architecture, a typical multi-peak powervoltage curve appears due to the effect of bypass diodes. And losses caused by the bypass diodes are significant. In contrast, DPP not only yields higher output power, but also results in a convex power-voltage curve of the PV module, with one unique maximum power point (MPP) voltage that is close to the nominal value [1]. As a result, the MPP could easily be tracked by the module-level converter/inverter with some traditional MPP tracking (MPPT) methods $[2,3]$.

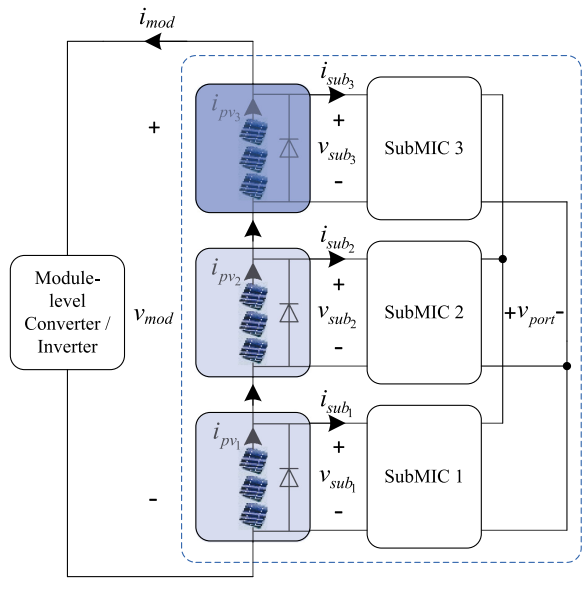

(a)

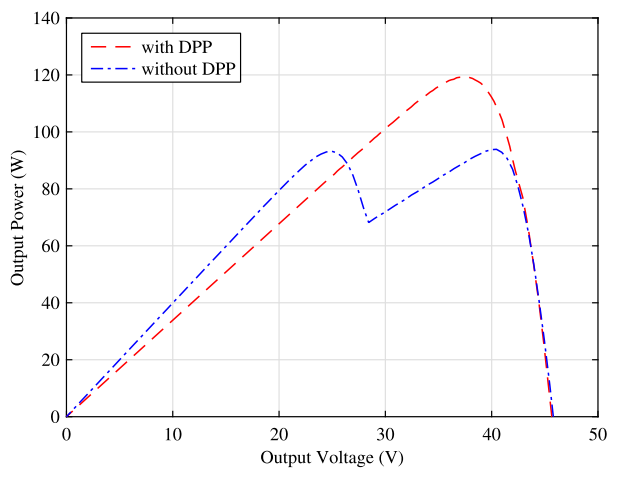

(b)

Fig. 1. (a) The structure of the DPP isolated-port PV architecture.

(b) Power-voltage curves of a mismatched PV module. 
Compared with the module-level [4] or submodule-level DC optimizers [5], no power is processed by the subMICs when mismatch disappears, thus great insertion losses are eliminated using this architecture. Compared with other DPP architectures, this isolated-port DPP architecture processes less power to keep the PV module working in the vicinity of MPP [6]. And the control of each subMIC is much simpler, without any current sensing performed.

To facilitate the control design of each subMIC, the dynamic model of the DPP isolated-port architecture should be developed. The small-signal model of this architecture was first developed in [1] by applying Middlebrook's extra element theorem. It provides a simple control strategy for the realization of the system, but the dynamics of the subMICs are linearized by look-up tables. Another small-signal model was proposed in [7] by using the method of perturbation and linearization, but the assumption of negligible current ripples is not satisfied in the dual active bridge (DAB) converter since its inductor current is pure ac. Besides, the interactions of the subMICs at the isolated port are both ignored in the above two works. Up to now, no in-depth analyses on the dynamic characteristics of the DPP isolatedport architecture have been presented in literatures.

The purpose of this work is to develop a small-signal model of the DPP isolated-port PV architecture by using discrete-time modeling methods $[8,9,10]$ and considering the interactions among different subMICs. The DPP PV architecture in this work is designed based on DAB converters considering their capabilities of isolation, bidirectional power transfer, and inherent merit of high power efficiency [11].

\section{The small-signal model of the DPP PV architecture}

A simplified block diagram of the small-signal model of the DPP PV architecture is shown in Fig. 2, where variables with hat indicate the small signals of the corresponding variables. As shown in the picture, the DPP architecture can be divided into three parts: (a) the module-level converter/inverter, (b) PV submodules, and (c) subMICs.

(a) The closed-loop input impedance of the module-level converter/inverter is denoted as $Z_{\text {mod }}$. The complete derivation of it depends on the specific regulation mode of the converter/inverter [12].

(b) The small-signal equivalent circuit of each PV submodule is represented as a current source $\hat{i}_{g 0_{i}}$ paralleled with a resistance $R_{g_{i}}$, where subscript $i$ denotes the index of the submodule. $R_{g_{i}}$ is obtained by the linearization of the nonlinear current-voltage curve of the PV submodule at its steady-state operating point.

(c) Each subMIC is modeled as a two-port network, with $\hat{i}_{g_{i}}$ and $\hat{i}_{\text {out }}$ modeled as the input variables, $\hat{v}_{\text {sub }_{i}}$ and $\hat{v}_{\text {out }_{i}}$ as the output variables. These four variables can be used as the interfaces to interact with the rest of the system. Therefore, each subMIC could be decoupled from the system and modeled independently. Besides, $R_{\text {in }}$ and $R_{\text {out }}$ represent the wire resistance at the input and output of each subMIC. 


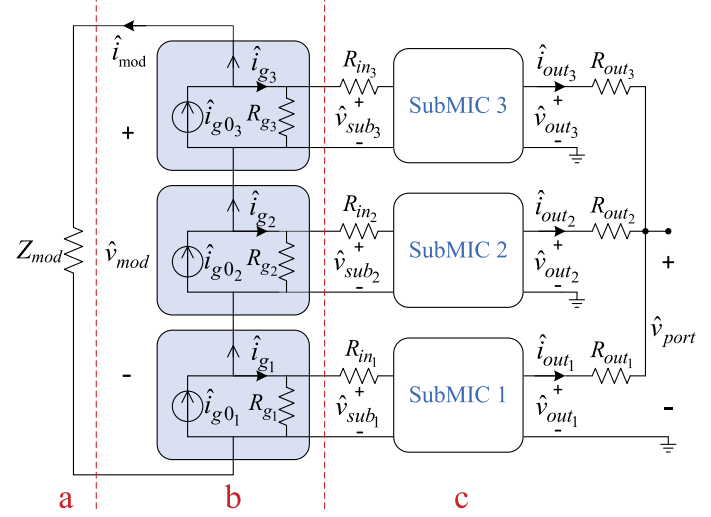

Fig. 2. Simplified block diagram of the small-signal model of the DPP isolated-port PV architecture.

\subsection{Discrete-time modeling of the decoupled DAB subMICs}

The power stage of the designed DAB subMIC is shown in Fig. 3(a). Two DC blocking capacitors $C_{b_{1,2}}$ are connected to both sides of the transformer to remove the voltage-second mismatches. The DAB subMICs are controlled by the phaseshift modulation at a fixed switching frequency of $100 \mathrm{KHz}$. A relatively large tank inductance $L_{t}$ is chosen so as to extend the range of zero voltage switching, and to increase the resolution of the digital phase shift.

Key time-domain waveforms of the DAB subMIC when power is transferred from primary side to secondary side are illustrated in Fig. 3(b), where $v_{p}(t)$ and $v_{S}(t)$ are the output voltages of the primary and secondary bridges, $i_{L}(t)$ is the inductor current. Zero voltage switching transitions are assumed to be instantaneous since a relatively low switching frequency is adopted. Thus each switching period could be divided into 4 subintervals as labeled, and the converter operates symmetrically every 2 subintervals.

In each subinterval, the subMIC circuit is linear, time-invariant, with the corresponding state-space description shown as follow

$$
\dot{x}_{i}(t)=A_{i, k} x_{i}(t)+B_{i, k} u_{i}(t)
$$

where $x_{i}(t)=\left[\begin{array}{lll}i_{L_{i}} & v_{\text {sub }} & v_{\text {out }}\end{array}\right]^{T}$ is the state vector, $u_{i}(t)=\left[\begin{array}{ll}i_{g_{i}} & i_{\text {out }}\end{array}\right]^{T}$ is the disturbance vector, subscripts $i$ and $k$ denote the index of the submodule and subinterval respectively. Since all derivations in this subsection are focused on the $i$ th subMIC,

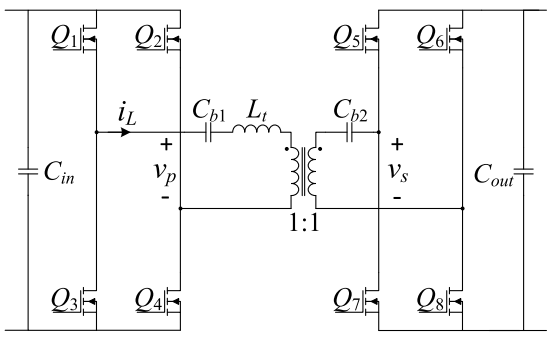

(a)

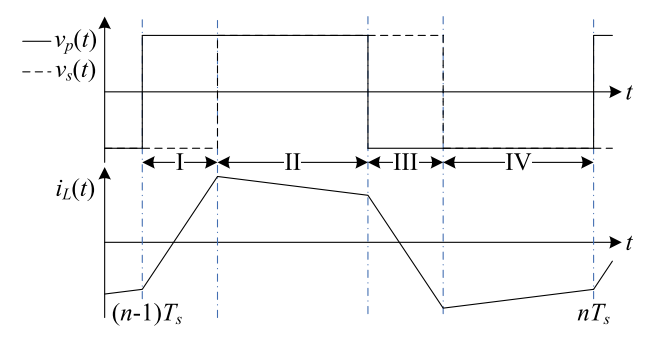

(b) 
subscript $i$ is omitted for simplification. The solution to equation (1) in each subinterval is then given by

$$
x(t)=e^{A_{k} t} x_{0}+\left(e^{A_{k} t}-I\right) A_{k}^{-1} B_{k} u(t)
$$

where $x_{0}$ is the initial condition of the state vector $x(t)$. By using the first-order Taylor series expansion $e^{A_{k} t}=I+A_{k} t$, Equation (2) could be linearized as follow

$$
x(t)=\left(I+A_{k} t\right) x_{0}+B_{k} t u(t)
$$

To facilitate the derivation of coefficients $A_{k}$ and $B_{k}$, equivalent circuits of the DAB subMIC corresponding to subintervals I-II are depicted in Fig. 4, where equivalent resistance $R_{e}$ is the sum of MOSFETs on resistance, transformer ac resistance, and inductor ac resistance. $R_{g}$ is also included in the subMIC modeling considering its direct effect on the input of its corresponding subMIC, whereas $R_{\text {in }}$ is neglected since it's too small compared to $R_{g}$. The effects of DC blocking capacitances $C_{b_{1,2}}$ and transformer magnetizing inductance $L_{m}$ on converter dynamics are ignored here.

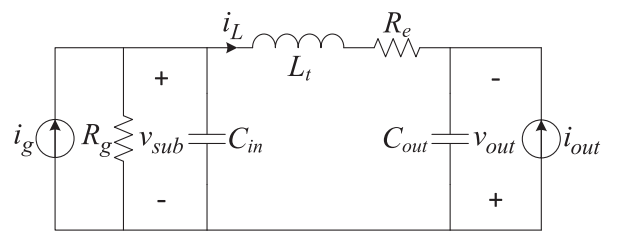

(a)

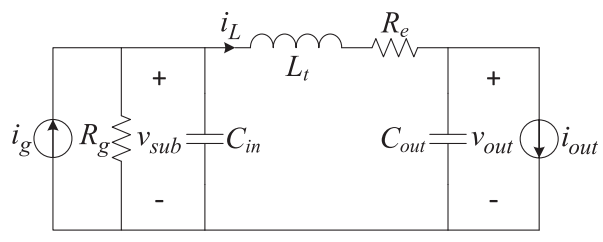

(b)

Fig. 4. Equivalent circuits of the decoupled DAB subMIC in subintervals (a) I and (b) II.

The resulting matrix coefficients $A_{1}, B_{1}$ and $A_{2}, B_{2}$ that correspond to subintervals I and II are obtained as below, while coefficients for subintervals III-IV can be easily obtained the same way.

$$
\begin{gathered}
A_{1}=\left[\begin{array}{ccc}
\frac{-R_{e}}{L_{t}} & \frac{1}{L_{t}} & \frac{1}{L_{t}} \\
\frac{-1}{C_{\text {in }}} & \frac{-1}{R_{g} C_{\text {in }}} & 0 \\
\frac{-1}{C_{\text {out }}} & 0 & 0
\end{array}\right] B_{1}=\left[\begin{array}{cc}
0 & 0 \\
\frac{1}{C_{\text {in }}} & 0 \\
0 & \frac{-1}{C_{\text {out }}}
\end{array}\right] \\
A_{2}=\left[\begin{array}{ccc}
\frac{-R_{e}}{L_{t}} & \frac{1}{L_{t}} & \frac{-1}{L_{t}} \\
\frac{-1}{C_{\text {in }}} & \frac{-1}{R_{g} C_{\text {in }}} & 0 \\
\frac{1}{C_{\text {out }}} & 0 & 0
\end{array}\right] \quad B_{2}=\left[\begin{array}{cc}
0 & 0 \\
\frac{1}{C_{\text {in }}} & 0 \\
0 & \frac{-1}{C_{\text {out }}}
\end{array}\right]
\end{gathered}
$$

Based on the discrete-time modeling approaches for digitally controlled converters $[8,9,10]$, the small-signal deviation of the state vector sampled at $n T_{s}$ should be a linear combination of the small-signal state vector $\hat{x}$, small perturbation of phase shift $\hat{\phi}$, and small-signal disturbance vector $\hat{u}$. 


$$
\hat{x}[n]=\alpha \hat{x}[n-1]+\beta \hat{\phi}[n-1]+\gamma \hat{u}[n-1]
$$

When only the effect of state perturbation $\hat{x}[n-1]$ is considered, $\phi$ and $u(t)$ are assumed to be constant. The resulting state propagation coefficient $\alpha$ could be derived by applying equation (2) consecutively in each subinterval

$$
\alpha=e^{A_{4} t_{4}} e^{A_{3} t_{3}} e^{A_{2} t_{2}} e^{A_{1} t_{1}}
$$

Similarly, the disturbance-to-state coefficient $\gamma$ could be obtained by applying equation (2) or (3) consecutively in each subinterval

$$
\gamma=e^{A_{4} t_{4}} e^{A_{3} t_{3}} e^{A_{2} t_{2}} B_{1} t_{1}+e^{A_{4} t_{4}} e^{A_{3} t_{3}} B_{2} t_{2}+e^{A_{4} t_{4}} B_{3} t_{3}+B_{4} t_{4}
$$

Fig. 5 shows the effect of phase-shift perturbation $\hat{\phi}[n-1]$ on the state vector $x(t)$, where the waveform of inductor current $i_{L}(t)$ is used representing $x(t)$. As shown, the state vector $x(t)$ is perturbed by $\hat{\phi}[n-1]$ at the two modulation edges of the phase shift. Two resulting linearized state perturbations can be obtained from equation (3) as the difference between state propagations in previous and next subintervals.

$$
\begin{aligned}
& \hat{x}_{p_{1}}[n-1]=\left(A_{1}-A_{2}\right) \hat{t}_{\phi} X_{p_{1}}+\left(B_{1}-B_{2}\right) \hat{t}_{\phi} U \\
& \hat{x}_{p_{2}}[n-1]=\left(A_{3}-A_{4}\right) \hat{t}_{\phi} X_{p_{2}}+\left(B_{3}-B_{4}\right) \hat{t}_{\phi} U
\end{aligned}
$$

State perturbations $\hat{x}_{p_{1}}[n-1]$ and $\hat{x}_{p_{2}}[n-1]$ then propagate to $n T_{s}$ in the same manner as given in equation (6). Thus the control-to-state coefficient $\beta$ can be derived as follow

$$
\beta=e^{A_{4} t_{4}} e^{A_{3} t_{3}} e^{A_{2} t_{2}}\left(A_{1}-A_{2}\right) X_{p 1} \frac{T_{s}}{2 \pi}+e^{A_{4} t_{4}}\left(A_{3}-A_{4}\right) X_{p 2} \frac{T_{s}}{2 \pi}
$$

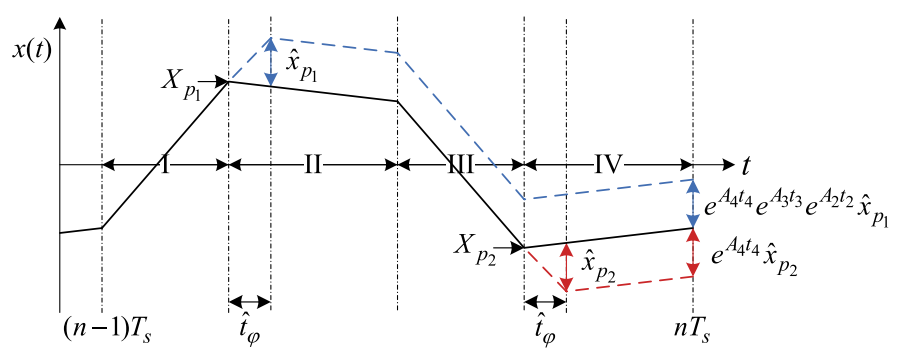

Fig. 5. Waveforms illustrating the linearized effect of the phase-shift perturbation $\hat{\phi}$ on state vector $x(t)$.

Substituting (6), (7) and (9) into equation (5), the standard Z-transform of (5) gives the desired discrete-time transfer functions of control-to-voltages $G_{\hat{v}_{i}} / \hat{\phi}_{i}(z)$ and disturbance-to-voltages $G_{\hat{v}_{i}} / \hat{u}_{i}(z)$, where $\hat{v}_{i}=\left[\hat{v}_{\text {sub }} \hat{v}_{\text {out }}\right]^{T}$.

$$
\begin{aligned}
& G_{\hat{v}_{i} / \hat{\phi}_{i}}(z)=\left[\begin{array}{lll}
0 & 1 & 0 \\
0 & 0 & 1
\end{array}\right]\left(z I-\alpha_{i}\right)^{-1} \beta_{i} \\
& G_{\hat{v}_{i} / \hat{u}_{i}}(z)=\left[\begin{array}{lll}
0 & 1 & 0 \\
0 & 0 & 1
\end{array}\right]\left(z I-\alpha_{i}\right)^{-1} \gamma_{i}
\end{aligned}
$$

Finally, the discrete-time small-signal model of the $i$ th decoupled DAB subMIC is obtained. 


$$
\left[\begin{array}{ll}
\hat{v}_{\text {sub }} & \hat{v}_{\text {out }}
\end{array}\right]^{T}=G_{\hat{v}_{i} / \hat{\phi}_{i}}(z) \hat{\phi}_{i}+G_{\hat{v}_{i} / \hat{u}_{i}}(z)\left[\begin{array}{ll}
\hat{i}_{g_{i}} & \hat{i}_{\text {out }_{i}}
\end{array}\right]^{T}
$$

\subsection{The small-signal model of the DPP architecture}

Taking the interactions among different subMICs into account, the complete smallsignal model of the DPP isolated-port PV architecture is depicted in Fig. 6, where $R_{\text {port }}=R_{\text {out }_{1}}\left\|R_{\text {out }_{2}}\right\| R_{\text {out }_{3}}$.

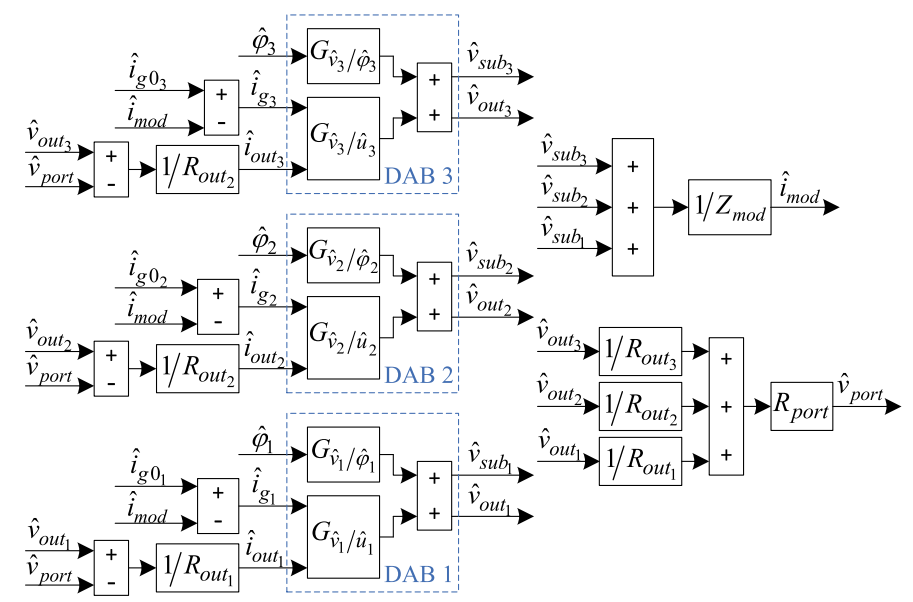

Fig. 6. Block diagram of the small-signal model of the DPP isolatedport PV architecture.

Consequently, transfer functions of control-to-submodule voltage $G_{\hat{v}_{s u b_{i}} / \hat{\phi}_{i}}(z)$ and control-to-output voltage $G_{\hat{\text { out }}_{i} / \hat{\phi}_{i}}(z)$, which model the effect of the phase-shift perturbation $\hat{\phi}_{i}$ on its corresponding submodule voltage and output voltage, could be derived.

$$
\begin{gathered}
G_{\hat{v}_{s u b_{i}} / \hat{\phi}_{i}}(z)=\frac{\hat{v}_{\text {sub }_{i}}}{\hat{\phi}_{i}} \mid \begin{array}{l}
\hat{\phi}_{j}=0, \forall j=1,2,3, j \neq i \\
\hat{i}_{g_{0} 0_{m}}=0, \forall m=1,2,3
\end{array} \\
G_{\hat{v}_{\text {out }} / \hat{\phi}_{i}}(z)=\frac{\hat{v}_{\text {out }_{i}}}{\hat{\phi}_{i}} \mid \begin{array}{l}
\hat{\phi}_{j}=0, \forall j=1,2,3, j \neq i \\
\hat{i}_{g_{m}}=0, \forall m=1,2,3
\end{array}
\end{gathered}
$$

In this work, transfer functions (12) and (13) are obtained numerically using the linear analysis tool in Matlab, as too many feedback loops need to be taken into account for the direct algebraic derivation.

Bode plots of $G_{\hat{v}_{\text {sub }} / \hat{\phi}_{1}}(z)$ and $G_{\hat{v}_{\text {out }} / \hat{\phi}_{1}}(z)$ are shown in Fig. 7, which correspond to three mismatch conditions when PV submodule 3 is mismatched by $10 \%, 50 \%$ and $90 \%$. These Bode plots are also verified by circuit simulation results using Plecs. As shown, the frequency responses of the analytical small-signal model are well matched to the simulation results. Steady-state parameters used for the analytical model and circuit simulation are:

1) $10 \%$ mismatch: $I_{p v_{1,2}}=3 \mathrm{~A}, I_{p v_{3}}=2.7 \mathrm{~A}, V_{s u b_{1,2,3}}=12 \mathrm{~V}, R_{g_{1,2,3}}=4.35 \Omega$.

2) $50 \%$ mismatch: $I_{p v_{1,2}}=3 \mathrm{~A}, I_{p v_{3}}=1.5 \mathrm{~A}, V_{s u b_{1,2,3}}=12 \mathrm{~V}, R_{g_{1,2,3}}=5.05 \Omega$.

3) $90 \%$ mismatch: $I_{p v_{1,2}}=3 \mathrm{~A}, I_{p v_{3}}=0.3 \mathrm{~A}, V_{s u b_{1,2,3}}=12 \mathrm{~V}, R_{g_{1,2,3}}=6.04 \Omega$. 
$I_{p v_{i}}$ is the submodule output current. $R_{g_{i}}$ is obtained by the linearization of the current-voltage curve of the Conergy S175MU PV submodule at its specific steadystate operating point. The small-signal closed-loop input impedance $Z_{\text {mod }}$ of the module-level converter/inverter depends on its regulation mode. It could be approximated as a small-signal zero resistance when the converter/inverter works in the voltage-regulation mode. However, since this work is focused on the modeling of the DPP architecture, the module-level converter/inverter was not designed. Thus $Z_{\text {mod }}$ is replaced by a resistance $R_{\text {mod }}$ which sets the module voltage to $V_{\text {mod }} \approx 36 \mathrm{~V}$.

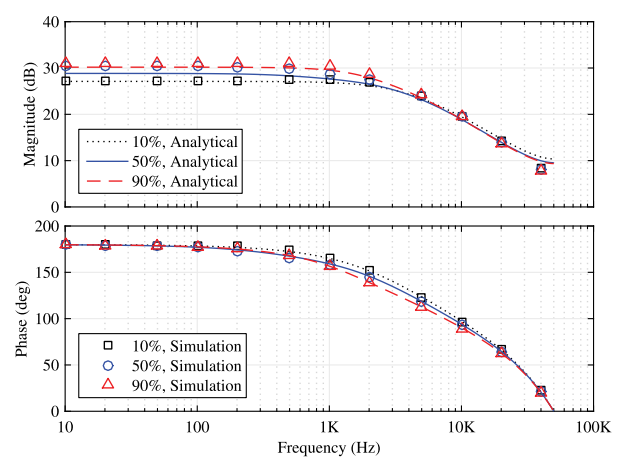

(a)

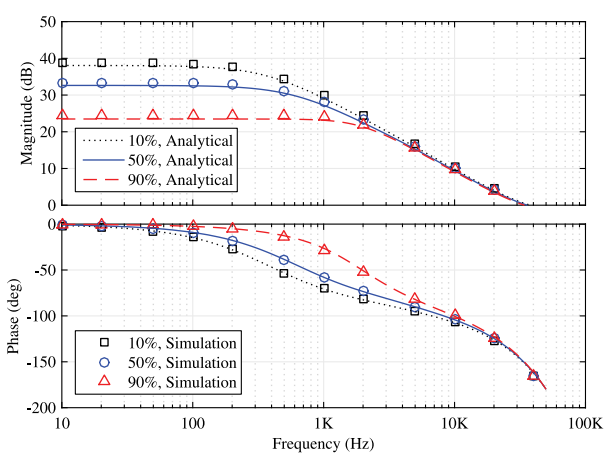

(b)

Fig. 7. Bode plots of (a) $G_{\hat{v}_{s u b_{1}} / \hat{\phi}_{1}}(z)$ and (b) $G_{\hat{v}_{o u t_{1}} / \hat{\phi}_{1}}(z)$ under three different mismatch conditions.

\subsection{Discrete compensator design for the subMICs}

The block diagram of the control loop for each subMIC is shown in Fig. 8. In order to equalize the submodule voltage to the isolated-port voltage, the error signal $\hat{e}_{i}$ between the subMIC output voltage $\hat{v}_{\text {out }}$ and the input voltage $\hat{v}_{\text {sub }}$ is used as the feedback variable, and zero voltage error is set as the reference. The effect of quantization and sampling delay is not taken into account in the current work.

The resulting transfer function of control-to-voltage error $G_{\hat{e}_{i} / \hat{\phi}_{i}}(z)$ is obtained

$$
G_{\hat{e}_{i} / \hat{\phi}_{i}}(z)=\frac{\hat{v}_{\text {out }_{i}}-\hat{v}_{\text {sub }}}{\hat{\phi}_{i}}=G_{\hat{v}_{\text {out }} / \hat{\phi}_{i}}(z)-G_{\hat{v}_{\text {sub }} /} / \hat{\phi}_{i}(z)
$$

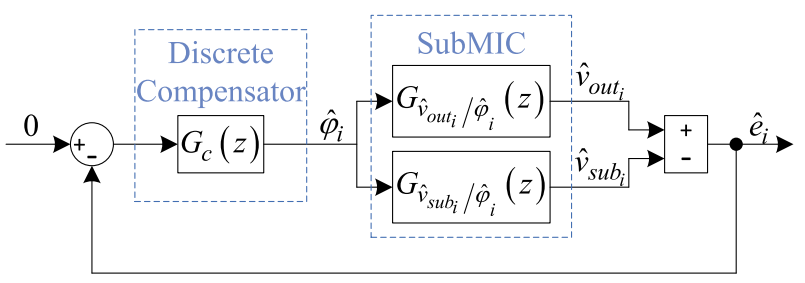

Fig. 8. Control block diagram for each subMIC.

Bode plots of $G_{\hat{e}_{i} / \hat{\phi}_{i}}(z)$ when the system works under the three aforementioned mismatch conditions, as well as their simulation verifications, are shown in 
Fig. 9(a), where parameters used are exactly the same as in Fig. 7. While the magnitude and frequency responses of $G_{\hat{v}_{\text {sub }} / \hat{\phi}_{i}}(z)$ and $G_{\hat{v}_{\text {out }} / \hat{\phi}_{i}}(z)$ are affected by different mismatch conditions, $G_{\hat{e}_{i}} \hat{\phi}_{i}(z)$ are not affected obviously. Therefore, a simple discrete PI compensator $G_{c}(z)$ of the form (15) could be employed to regulate the voltage error $\hat{e}_{i}$.

$$
G_{c}(z)=K_{p}\left(1+\frac{T_{s}}{T_{i}} \frac{z}{z-1}\right)
$$

The resulting loop gain of each subMIC is given by

$$
T_{i}(z)=G_{c}(z) G_{\hat{e}_{i} / \hat{\phi}_{i}}(z)
$$

Fig. 9(b) shows three bode plots of $T_{i}(z)$ that correspond to $G_{\hat{e}_{i} / \hat{\phi}_{i}}(z)$. As shown, when PV module works under different mismatch conditions, the crossover frequencies of the control loops are approximately $2 \mathrm{kHz}$, and the phase margin is always above $65^{\circ}$.

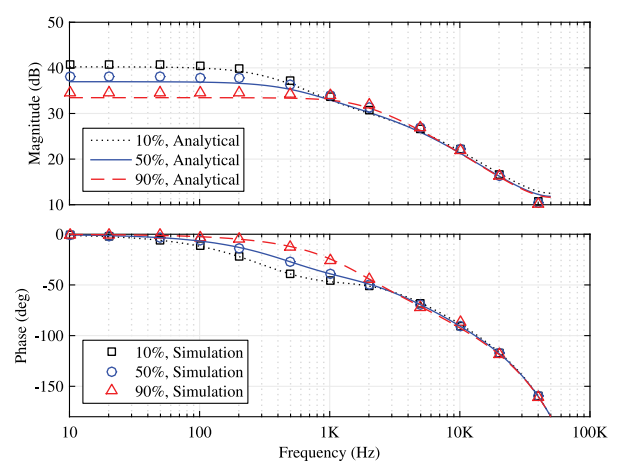

(a)

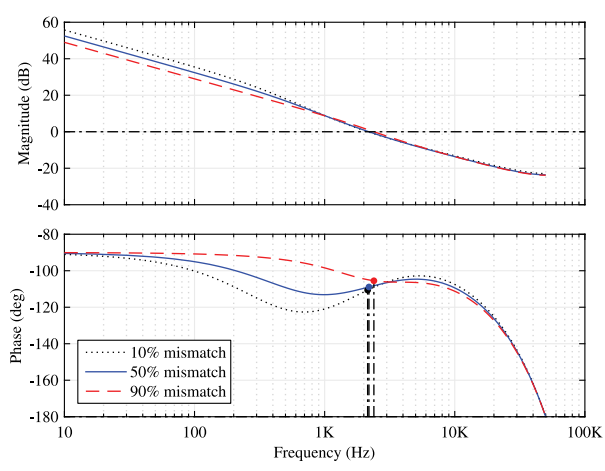

(b)

Fig. 9. Bode plots (a) $G_{\hat{e}_{i} / \hat{\phi}_{i}}(z)$ and (b) $T_{i}(z)$.

\section{Experimental results}

To verify the proposed small-signal model and control design, a prototype consisting of three $50 \mathrm{~W}$ DAB subMICs was constructed. Key components and circuit parameters are given in Table I. The prototype is shown in Fig. 10(a). It was designed with the dimension of $10 \mathrm{~cm}$ by $15 \mathrm{~cm}$, which can be fitted into the junction box at the back of the PV module. In the prototype, three same DAB subMICs are designed on the same board with the outputs connected in parallel as the isolated port. Three low-power micro-controllers MSP430F5172 implement the sampling and PI compensation for each subMIC. The indoor experimental setup is shown in Fig. 10(b), subMICs are attached to their corresponding submodules in a Conergy S175MU PV module. Conergy S175MU is a 72-cell module consisting of three 24-cell submodules, with the nominal MPP being at $V_{m p p}=36 \mathrm{~V}, P_{m p p}=$ $175 \mathrm{~W}$. 
Table I. Key components and circuit parameters of the DAB subMIC prototype

\begin{tabular}{cccccccc}
\hline$Q_{1} \sim Q_{8}$ & $V_{\text {in }}$ & $V_{\text {out }}$ & $C_{\text {in }}$ & $C_{\text {out }}$ & $L_{t}$ & $L_{m}$ & $C_{b_{1,2}}$ \\
\hline IRF8707 & {$[8,25] \mathrm{V}$} & {$[8,25] \mathrm{V}$} & $10 \mu \mathrm{F}$ & $10 \mu \mathrm{F}$ & $2.7 \mu \mathrm{H}$ & $150 \mu \mathrm{H}$ & $47 \mu \mathrm{F}$ \\
\hline
\end{tabular}

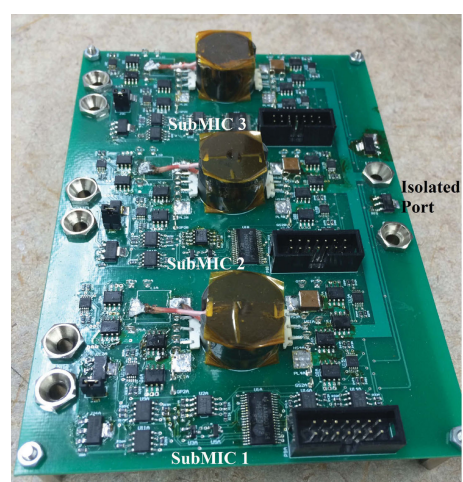

(a)

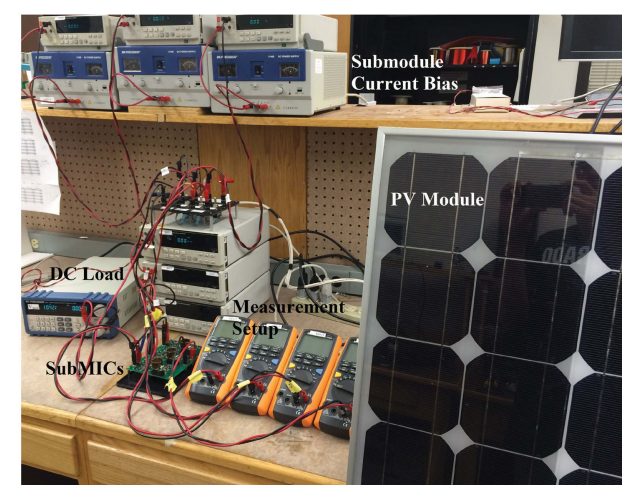

(b)

Fig. 10. (a) Prototype of the DAB subMICs. (b) Indoor experimental setup.

\subsection{Steady-state results}

In order to verify the main features of the DPP isolated-port PV architecture, powervoltage curves of a mismatched PV module with and without the DPP architecture were measured. Each submodule is connected in parallel with a voltage source working in the current-limit mode, which could emulate different submodule shortcircuit currents $i_{s c_{i}}$. An electronic load was connected to the output of the module. It was programmed to perform a voltage sweep while measuring the module output power.

Experimental power-voltage curves are shown in Fig. 11, where $i_{s c_{1,2}}=4 \mathrm{~A}$, $i_{s c_{3}}=2.5 \mathrm{~A}$, which means submodule 3 was mismatched by $3 / 8$. Without the DPP architecture, a typical multi-peak power-voltage curve appears due to the effect of bypass diodes, with the MPP being at $40.5 \mathrm{~V}, 93.9 \mathrm{~W}$. In contrast, DPP results in a convex power-voltage curve when the module voltage $v_{\text {mod }}>24 \mathrm{~V}$, with a single MPP being at $37.5 \mathrm{~V}, 119.5 \mathrm{~W}$, which is quite close to the ideal value. The ideal curve is measured when $i_{s c_{1,2,3}}$ are all set to $3.5 \mathrm{~A}$, which is the average value of the mismatched $i_{s c_{1,2,3}}$. In this case, no mismatch exists and the maximum available power of the PV module is equal to the mismatched case. Note that these subMICs are designed working in the range of $[8,25] \mathrm{V}$, thus the subMICs are shut down and bypass diodes take effect when $v_{\text {mod }}<24 \mathrm{~V}$. However, the whole power-voltage curve of the PV module with DPP could be verified by using external power supplies as [1] did if needed. 


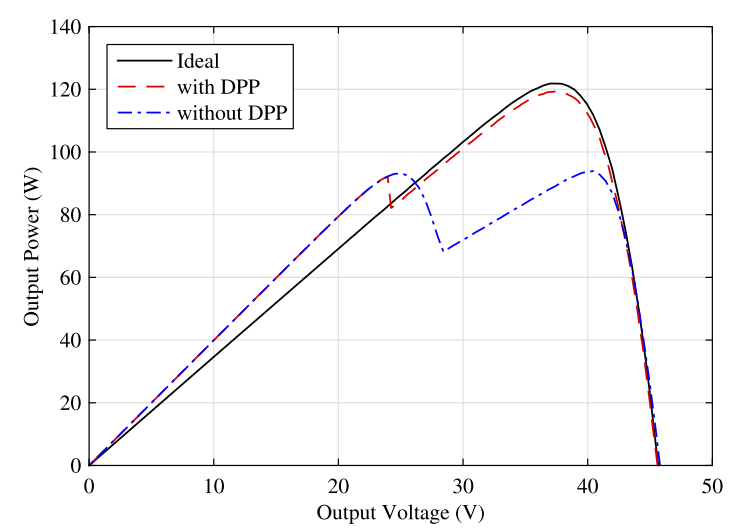

Fig. 11. Experimental power-voltage curves of a mismatched PV module.

\subsection{Dynamic responses}

To verify the analytical small-signal model of the DPP architecture, bode plots of $G_{\hat{v}_{s u b_{i}} / \hat{\phi}_{i}}(z)$ and $G_{\hat{v}_{\text {out }} / \hat{\phi}_{i}}(z)$ were verified by experiments. The experiment was implemented outdoors. PV submodule 3 was covered with a customized plastic film which could keep out $2 / 3$ of the solar irradiations. The output voltage of the PV module was adjusted by a rheostat.

The experiment was implemented in a two-step mode. In step 1, all the subMICs worked autonomously until a desired steady state was achieved: $V_{\text {sub } b_{1,2,3}}=12 \mathrm{~V}, I_{\text {mod }}=2.33 \mathrm{~A}$. In step 2, the PI compensators were stopped working, and some pre-programmed sinusoidal perturbations of several typical frequencies were superposed on the phase shift $\phi_{1}$ of subMIC 1 . The waveforms of $v_{s u b_{1}}$ and $v_{\text {out }}$ were then captured and analyzed by an oscilloscope. Experimental results of the bode plots, together with the simulation results, are shown in Fig. 12. It can be seen that the bode plots of $G_{\hat{v}_{s u b_{1}} / \hat{\phi}_{1}}(z)$ and $G_{\hat{v}_{\text {out }} / \hat{\phi}_{1}}(z)$ are well matched to the simulation and experimental results.

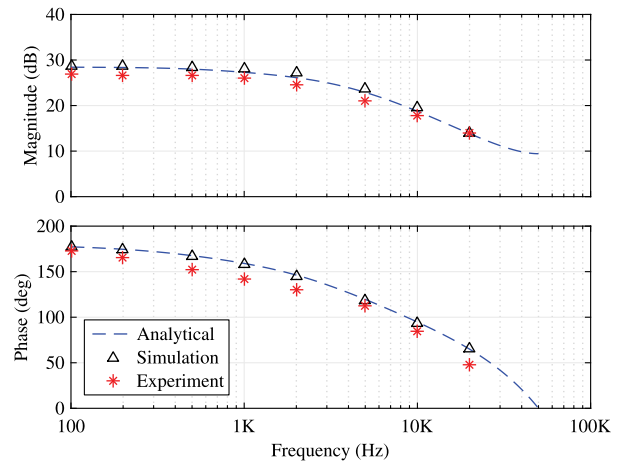

(a)

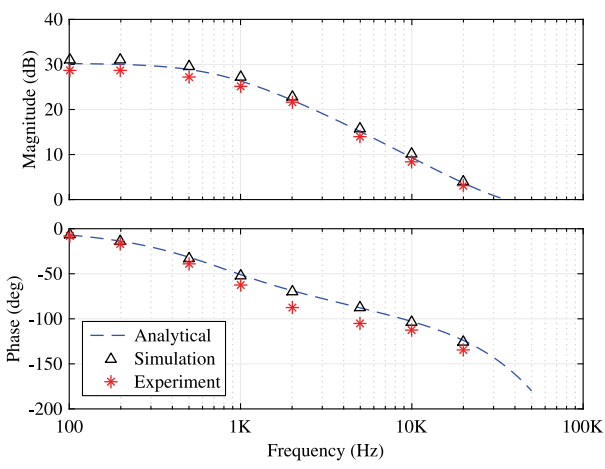

(b)

Fig. 12. Experimental magnitude and phase responses of (a) $G_{\hat{v}_{\text {sub }} / \hat{\phi}_{1}}(z)$ and (b) $G_{\hat{\text { vut }}_{1} / \hat{\phi}_{1}}(z)$ when submodule 3 is mismatched by $2 / 3$.

To verify the control design of the system, experiments of step responses were carried out indoors. In this experiment, submodule short-circuit currents $i_{s c_{1,2,3}}$ were 
set by the voltage sources as aforementioned. An electronic load was connected in parallel with submodule 3 to implement the step variation of $i_{s c_{3}}$. A rheostat was still used to regulate the PV module voltage.

The experiment was implemented as follows. At first, $i_{s c_{1,2,3}}$ were set to $3 \mathrm{~A}$, and $V_{\text {mod }}$ was set to $36 \mathrm{~V}$. At $t=20 \mathrm{~ms}, i_{s c_{3}}$ was pulled down abruptly to $1 \mathrm{~A}$ using the electronic load, while $i_{s c_{1,2}}$ kept constant during the experiment. The step responses of submodule voltages $v_{s u b_{1,2,3}}$ and subMICs input currents $i_{s u b_{1,2,3}}$ are shown in Fig. 13(a) and Fig. 13(b). It can be seen that the voltages and currents exhibit a smooth response, and converge to the steady state in less than $3 \mathrm{~ms}$.

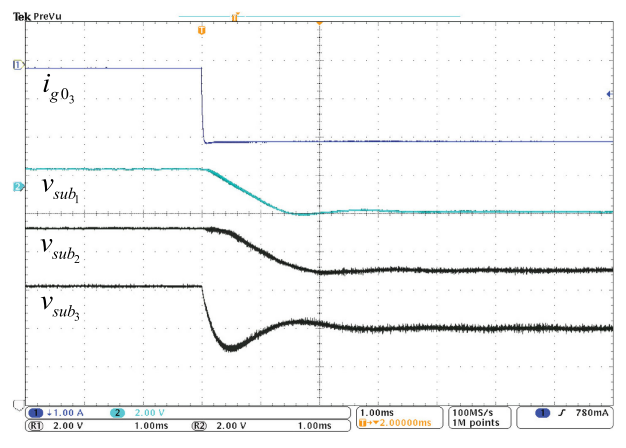

(a)

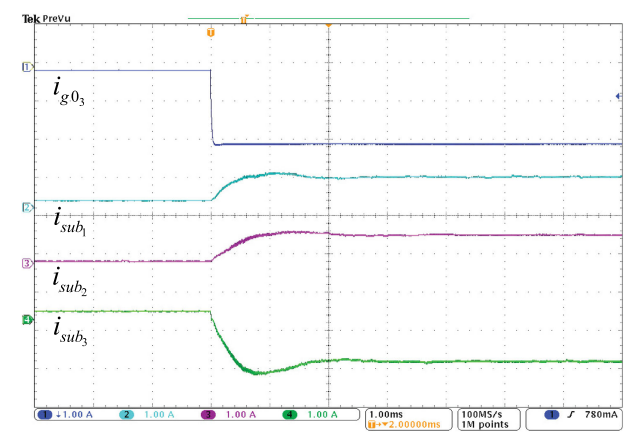

(b)

Fig. 13. Experimental step responses of (a) submodule voltages $v_{s u b_{1,2,3}}$ and (b) subMICs input currents $i_{s u b_{1,2,3}}$ to a step disturbance of $-2 \mathrm{~A}$ on $i_{s c_{3}}$.

\section{Conclusion}

A small-signal model of the DPP isolated-port PV architecture is developed through the discrete-time modeling method. With the help of the developed model, discrete compensator for the subMICs is designed accordingly. The validity of the modeling and control is verified by experimental results.

\section{Acknowledgments}

This work is supported by the Colorado Power Electronics Center (CoPEC) and China Scholarship Council (CSC). 